\title{
The flexibility of biobanking with the Bio-PIN system
}

\section{J.J. Nietfeld, Jeremy Sugarman and Jan-Eric Litton}

We thank Eric Vermeulen and colleagues for their recent correspondence in which they express their concerns about the Bio-PIN system that we described in our article (The Bio-PIN: a concept to improve biobanking. Nature Rev. Cancer 11, 303-308 (2011)) ${ }^{1}$. While we welcome broad discussion of the system, a crucial first step is that it is properly understood. Vermeulen et al. (The BIO-PIN paradigm: 'access to' or 'return of' results? Nature Rev. Cancer 4 Nov 2011 (doi:10.1038/ nrc3022-c1) $)^{2}$ assume that that the Bio-PIN system uses genotype identification. However, regardless of whether the Bio-PIN is based on short tandem repeats or on single nucleotide polymorphisms, the nucleotides involved are not part of any gene and are therefore not part of any genotype. Furthermore, the Bio-PIN system is anonymous, thus biological samples in that system cannot be used to assess the identity of the individuals that they originated from ${ }^{3}$.

In addition, Vermeulen et al. assume that individual donors cannot be directly approached to provide additional information to a biobank that uses the Bio-PIN system. However, this 'flaw' seems misplaced. For example, patients could register the fact that they are biobank donors in the medical facilities from which they seek care. When visiting these facilities those patients could be asked to interact anonymously with the biobank. Then they could, for example, approve future research on their samples (or not), update information, such as lifestyle data, or agree with (residual) specimens and/or test results being sent to the biobank, as discussed in our article ${ }^{1}$. It seems entirely plausible that when biobank donors consider the benefits of having their own electronic personal health record in their 'biobank account', they will be encouraged to regularly update their information.

While the Dutch voluntary Code is of obvious importance, its global implications at this point in time are arguably limited. Furthermore, on translation from Dutch to English, the Code does not indicate that biobanks, in the case of residual specimens, should always be able to seek contact with donors. In general, if patients' specimens are stored for their future care, then they are governed under the norms of standard health care procedures, which include the possibility of contacting those patients. However, if the part of a specimen that remains after a diagnostic test is stored and is no longer necessary for future patient care and is going to be used for research, it is no longer governed by these same provisions. Accordingly, such residual material could then be made anonymous and the Bio-PIN system applied.

The Bio-PIN system is very flexible. It enables donors and biobanks to communicate in a secure way, exchanging information, as discussed in our article ${ }^{1}$. This fully allows donors and the biobank to agree how and in what form data and results will be made available to donors. The data interpretation and counselling that the biobank might offer would be dependent on the governance of the biobank and the particular information that is available. How biobanks arrange these types of services might become an important factor in determining whether donors become participants who wish to invest more samples and/or data in the advancement of medical science.

How the various aspects of the Bio-PIN system will ultimately work in practice can hopefully be investigated in a pilot project.

J.J. Nietfeld is at the University Medical Center Utrecht, Department of Pathology, Utrecht 3584CX, The Netherlands.

Jeremy Sugarman is at the Berman Institute of Bioethics and School of Medicine, Johns Hopkins University, Baltimore 21205, Maryland, USA.

Jan-Eric Litton is at the Department of Medical Epidemiology and Biostatistics, Karolinska Institutet, Stockholm SE-171 77, Sweden.

Correspondence to J.J.N. e-mail: J.J.Nietfeld@umcutrecht.n! doi: $10.1038 / n r c 3022-c 2$

Nietfeld, J. J., Sugarman, J. \& Litton, JE. The Bio-PIN: a concept to improve biobanking. Nature Rev. Cancer 11, 303-308 (2011).

2. Vermeulen, E., van der Valk, P., Koevoets, J. J. M. Cornel, M. C. \& Schmidt, M. K. The BIO-PIN paradigm: 'access to' or 'return of ' results? $4 \mathrm{Nov}$ 2011 (doi: 10.1038/nrc3022-c1)

3. Nietfeld, J. J. What is anonymous? EMBO reports $\mathbf{8}$ 518 (2007).

Competing interests statement

An international patent application has been filed by INTRESCO Ltd. J.J.N. has a potential future competing financial interest. 